\title{
Hepatic arterial infusion chemotherapy followed by sorafenib in patients with advanced hepatocellular carcinoma (HICS 55): an open label, non-comparative, phase II trial
}

Masahiro Hatooka ${ }^{1}$, Tomokazu Kawaoka', Hiroshi Aikata ${ }^{1 *}$, Yuki Inagaki ${ }^{1}$, Kei Morio ${ }^{1}$, Takashi Nakahara', Eisuke Murakami ${ }^{1}$, Masataka Tsuge ${ }^{1}$, Akira Hiramatsu' ${ }^{1}$, Michio Imamura', Yoshiiku Kawakami ${ }^{1}$, Kazuo Awai ${ }^{2}$, Keiichi Masaki ${ }^{3}$, Koji Waki $^{3}$, Hirotaka Kohno ${ }^{4}$, Hiroshi Kohno ${ }^{4}$, Takashi Moriya ${ }^{5}$, Yuko Nagaoki ${ }^{6}$, Toru Tamura ${ }^{6}$, Hajime Amano ${ }^{7}$, Yoshio Katamura ${ }^{7}$ and Kazuaki Chayama ${ }^{1,8,9}$

\begin{abstract}
Background: In patients with advanced hepatocellular carcinoma (HCC), evidence is unclear as to whether hepatic arterial infusion chemotherapy (HAIC) or sorafenib is superior. We performed a prospective, open-label, non-comparative phase II study to assess survival with HAIC or HAIC converted to sorafenib.

Methods: Fifty-five patients were prospectively enrolled. Patients received HAIC as a second course if they had complete response, partial response, or stable disease (SD) with an alpha fetoprotein (AFP) ratio $<1$ or a des- $\gamma$-carboxy prothrombin (DCP) ratio $<1$. Patients were switched to sorafenib if they had SD with an AFP ratio $>1$ and a DCP ratio $>1$ or disease progression. The primary endpoint was the 1-year survival rate. Secondary endpoints were the 2-year survival rate, HAIC response, survival rate among HAIC responders, progression-free survival, and adverse events.

Results: Of the 55 patients in the intent-to-treat population, the 1-year and 2-year survival rates were 64.0 and $48.3 \%$, respectively. After the first course of HAIC, one (1.8\%) patient showed complete response, 13 (23.6\%) showed partial response, $30(54.5 \%)$ had SD, and 10 (18.1\%) patients had progressive disease. Twenty-three patients (41.8\%) had SD with AFP ratios $<1$ or DCP ratios $<1$, and $7(12.7 \%)$ had SD with AFP ratios $>1$ and DCP ratios $>1$. Thirty-seven patients (68.5\%) were responders and 17 (30.9\%) were non-responders to HAIC. In responders, the 1-year and 2-year survival rates were 78 and $62 \%$, respectively.
\end{abstract}

Conclusion: Given the results of this study, this protocol deserves consideration for patients with advanced HCC. This trial was registered prospectively from December 12. 2012 to September 1. 2016.

Keywords: HCC, HAIC, Sorafenib, Tumor marker, RECIST

\footnotetext{
* Correspondence: aikata@hiroshima-u.ac.jp

${ }^{1}$ Department of Gastroenterology and Metabolism, Institute of Biomedical \&

Health Science, Hiroshima University, Hiroshima 734-8551, Japan

Full list of author information is available at the end of the article
}

(c) The Author(s). 2018 Open Access This article is distributed under the terms of the Creative Commons Attribution 4.0 International License (http://creativecommons.org/licenses/by/4.0/), which permits unrestricted use, distribution, and reproduction in any medium, provided you give appropriate credit to the original author(s) and the source, provide a link to the Creative Commons license, and indicate if changes were made. The Creative Commons Public Domain Dedication waiver (http://creativecommons.org/publicdomain/zero/1.0/) applies to the data made available in this article, unless otherwise stated. 


\section{Background}

Hepatocellular carcinoma (HCC) is the sixth most common cancer and the second leading cause of cancer-related mortality in the world. [1, 2]. Advances in technology have contributed to development of new diagnostic techniques such as ultrasonography, computed tomography, magnetic resonance imaging, and angiography. Similarly, new treatment modalities have been developed, including surgical resection, radiofrequency ablation [3], percutaneous ethanol injection, transcatheter arterial chemoembolization (TACE), and hepatic arterial infusion chemotherapy (HAIC), resulting in improved prognosis in HCC patients [4-12]. However, the survival rates are still poor for patients with advanced HCC with associated complications such as portal vein tumor thrombosis, and refractoriness to TACE.

Two phase III clinical trials of sorafenib for advanced HCC showed significant efficacy in terms of overall survival (OS) time compared with placebo [13, 14]. Based on these studies, sorafenib has become the standard of therapy for advanced HCC. Sorafenib is associated with extension of OS time by $2.3-2.8$ months and the improvement of response rate by $2.0-3.3 \%$. However, the survival advantage of sorafenib has been described as insufficient.

HAIC is widely used throughout Asia, especially in Japan. Several studies have shown the survival benefits of HAIC for advanced HCC free of extrahepatic metastasis (extrahepatic spread, or EHS), with response rates ranging from 20.8 to $52 \%$, and have shown that the median survival time (MST) in responders ranges from 17.6-40.7 months [11, 12, 15-18]. In most retrospective studies, the survival time was much better among responders than non-responders. Nevertheless, HAIC is not regarded as the standard of care for advanced HCC patients as no prospective randomized phase III trials have shown survival benefits in patients with advanced HCC.

Among responders, a better prognosis was expected with HAIC compared with sorafenib, while HAIC non-responders had a poor prognosis at 6 months in previous studies. Therefore, it is necessary to identify HAIC non-responders as early as possible.

In a previous study, we reported that patients showing either complete or partial response (CR and PR respectively) by the first course of HAIC had good prognoses, whereas patients with progressive disease [19] by the first course of HAIC had poor prognoses. However, we observed that the majority of patients had stable disease (SD) after the first course of HAIC. Furthermore, we reported that among patients determined to have SD based on the imaging response to the first course of HAIC, those with alpha fetoprotein (AFP) and des- $\gamma$-carboxy prothrombin (DCP) ratios $>1$ had significantly poorer survival times [20].That is, patients in whom AFP or DCP levels decreased had better prognoses than those in whom AFP or DCP levels increased. Therefore, we considered patients to be HAIC responders in the first course of HAIC when they showed CR, PR, or SD with decreased levels of AFP or DCP. We defined HAIC non-responders as either patients with PD or patients with SD who had increased levels of AFP and DCP after the first course of HAIC.

Few prospective studies of HAIC have been performed. No study protocols have been examined in which HAIC was continued only in responders while non-responders were switched to sorafenib where the outcome of the first course of HAIC was determined by early assessment of tumor markers and imaging responses. Therefore, we created a protocol in which HAIC was continued unless the outcome of therapy was non-response, and non-responders were then switched from HAIC to sorafenib.

\section{Methods}

\section{Study design}

The phase II HICS study (Hepatic Arterial Infusion Chemotherapy followed by Sorafenib) was a single-arm, prospective, open-label trial. In this study, the primary endpoint was the survival rate at 1 year. The secondary endpoints were the survival rate at 2 years, overall survival (OS), response to HAIC, survival rate according to HAIC response, progression-free survival (PFS), and adverse events (AEs). The primary endpoint, survival rate at 1 year, was defined as the probability of patients being alive 1 year after their first course of HAIC. OS was defined as the time from the start of the study treatment to the death due to any reason. PFS was defined as the time from the start of study treatment to the first documentation of objective tumor progression or to death due to any cause.

One month after the first course of HAIC, therapeutic efficacy was assessed by imaging studies and AFP/DCP. Results of imaging studies were assessed according to the Response Evaluation Criteria In Solid Tumors.

Safety assessments of the drugs included recording of AEs, changes in laboratory test results, physical examination, and vital signs. Adverse events associated with the drugs were those listed in the Common Terminology Criteria for Adverse Events (CTCAE) 4.0.

The study was registered with the University Hospital Medical Information Network Clinical Trials Registry as HICS 55, with the identifier number UMIN 000009094. The study was approved by the ethics committee and conducted in accordance with the Declaration of Helsinki. Informed consent was obtained from each patient.

\section{Patients}

Key inclusion criteria were as follows: minimum age of 20 years; life expectancy of at least 12 weeks at the pre-treatment evaluation; advanced HCC based on histological evidence via biopsy specimen or dynamic computed 
tomography or magnetic resonance imaging; not eligible for resection or local ablation therapy or TACE; at least 4 weeks since the last therapy for HCC; no prior sorafenib and HAIC treatment; no intrahepatic tumor that could affect patient prognosis; Eastern Cooperative Oncology Group performance status of 0 or 1; Child-Pugh score of 5,6 , or 7 ; and adequate bone marrow, liver, and renal function, as assessed by the following laboratory requirements: granulocyte count $\geq 3000 / \mathrm{mm}^{3}$, platelet count $\geq 50,000 / \mathrm{mm}^{3}$, hemoglobin $\geq 8.5 \mathrm{~g} / \mathrm{dL}$, total serum bilirubin $\leq 3 \mathrm{mg} / \mathrm{dL}$, serum albumin $\geq 2.8$, serum creatinine $\leq 1.5 \mathrm{mg} / \mathrm{dL}$, prothrombin consumption test $\geq 50 \%$, and amylase $\leq$ twice the upper limit of normal. Key exclusion criteria were as follows: other malignant disease, pregnancy or suspected pregnancy, severe infectious disease, history of severe allergy, severe renal function disease, severe allergy to 5-fluorouracil or cisplatin, severe bone marrow suppression, esophageal and/or gastric varices with a high risk of bleeding and clinically significant gastrointestinal bleeding, or serious hypertension. Patients who were unstable or whose safety or compliance in the study could be jeopardized based on the investigator's judgment were also excluded.

\section{Treatments}

Figure 1 illustrates the study schema. HAIC was administered as the first therapy. Within one month after HAIC administration, efficacy was assessed by imaging studies and AFP/DCP. Patients who showed CR or PR or SD with AFP ratio $<1$ or DCP ratio $<1$ were defined as responders. Patients who showed SD with AFP ratio $>1$ and DCP ratio $>1$ or PD [19] were defined as non-responders. Responders continued HAIC while non-responders were switched from HAIC to sorafenib. The therapeutic efficacy of sorafenib was assessed by imaging studies and AFP/ DCP one month after starting therapy. TACE was provided to partial and non-responders during this study.

\section{Hepatic arterial infusion chemotherapy}

Cisplatin was administered at a dose of $20 \mathrm{mg} / \mathrm{m}^{2} /$ day on days 1 and 8 , and fluorouracil was administered at a dose of $330 \mathrm{mg} / \mathrm{m}^{2} /$ day on days $1-5$ and 8-12 of every 28-day cycle, followed by 2 weeks off. HAIC was interrupted in patients who experienced hematologic and non-hematologic toxicities attributed to HAIC.

\section{Sorafenib}

Sorafenib $400 \mathrm{mg}$ bid was used for the treatment of patients who switched from HAIC. Sorafenib doses were adjusted, by interruption or reduction, in patients who experienced clinically significant hematologic or non-hematologic toxicities attributed to sorafenib. Sorafenib doses were reduced stepwise from $400 \mathrm{mg}$ twice daily to $400 \mathrm{mg}$ once daily to $400 \mathrm{mg}$ every other day to $200 \mathrm{mg}$ every other day as warranted. Stepwise increases were allowed after resolution of the AE. TACE, radiation therapy, and hepatectomy were allowed as additional therapies.

\section{Statistical analysis}

We assumed a threshold survival rate at 1 year of $45 \%$ with an expected survival rate at 1 year of $60 \%$ (0.1 $\alpha$-error and $0.1 \beta$-error). From these, we predicted that 46 patients would qualify and established a patient enrollment target of 55 assuming that $20 \%$ would be disqualified.

Statistical analysis was performed using SPSS (IBM, Armonk, NY, USA). Continuous variables are expressed

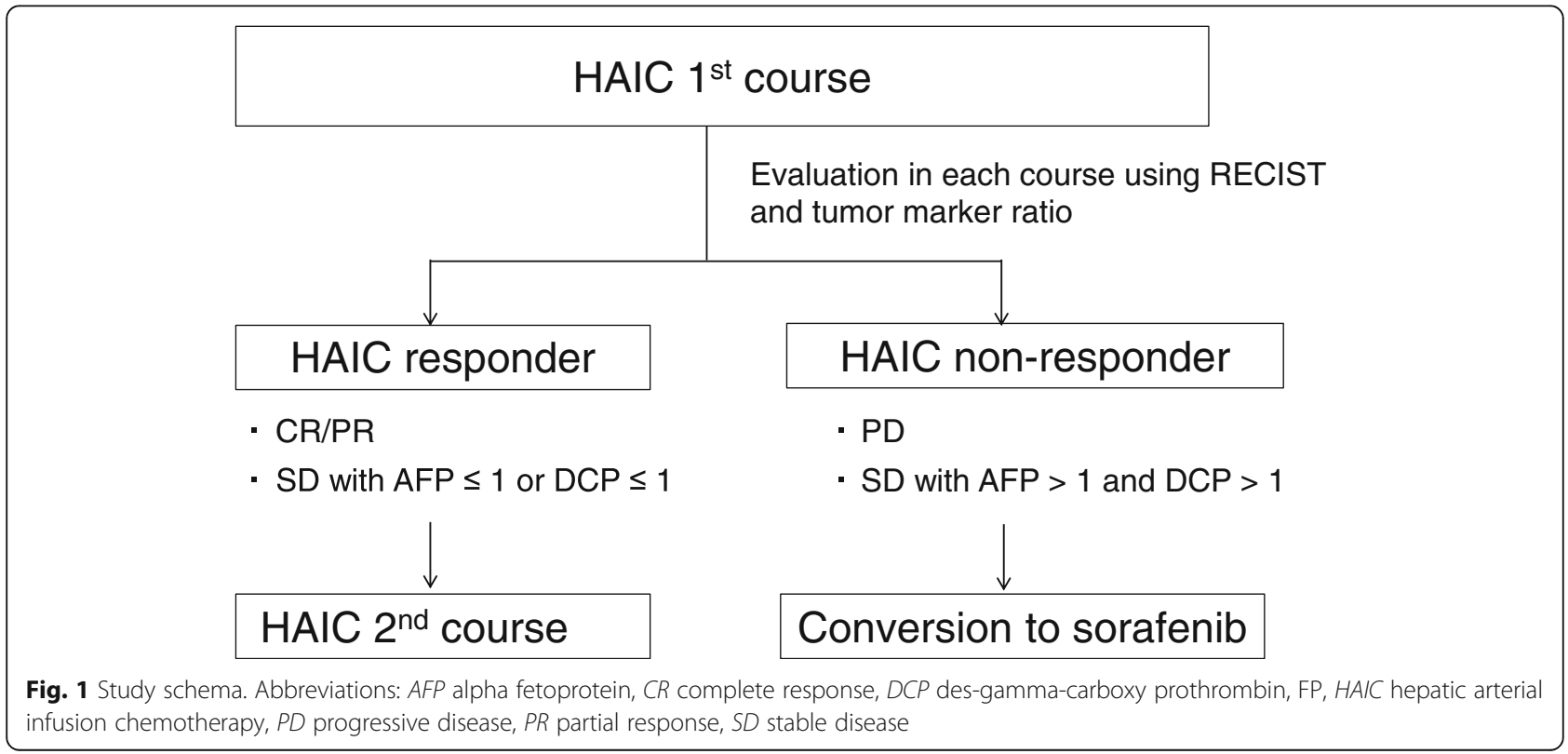


as medians and ranges, while categorical variables are expressed as counts or frequencies. Kaplan-Meier survival curves with log-rank tests were used for the analysis of OS. The statistical analysis was performed in September 2017. Differences between groups were examined for statistical significance using the Mann-Whitney U test, logistic regression test, or chi-square test as appropriate. The cumulative survival rate was calculated from the date of initiation of HAIC and assessed by the Kaplan-Meier life-table method. Differences between groups were evaluated by the log-rank test. For baseline characteristics such as performance status, age, stage of disease, and history of therapy, we calculated frequencies, averages, and medians to assess their distribution.

Variables that achieved statistical significance $(P<0.05)$ or marginal significance $(P<0.10)$ in the univariate analysis were entered into multiple logistic regression analysis to identify significant independent predictive responders.

Multivariate Cox proportional hazards regression was performed to assess the independent prognostic factors. For both univariate and multivariate analyses, all independent factors that demonstrated statistical significance as a predictor were analyzed using stepwise selection in the model. Hazard ratios and corresponding 95\% confidence intervals are reported.

\section{Results}

\section{Baseline characteristics}

Between December 2012 and October 2016, 55 patients with unresectable HCC were enrolled in this study at participating hospitals in the Hiroshima Liver study group. The median period of observation was 12.2 months with a range of 2.1 to 54.6 months. The data was last updated on September 2017.

Patient characteristics are listed in Table 1. The majority of study subjects were male, with a median age of 66 years. Among 29 patients who had Vp 3 and 4, 19 patients received three-dimensional conformal radiotherapy. Patients received HAIC therapy a median of two times (range: 0 to 11 times).

\section{Efficacy}

Figure 2 shows the flow of patients through the study. The number of responders was 37 patients (68.5\%), and the number of non-responders was 17 patients (30.9\%). Among the responders, 32 patients received a second course of HAIC. Five patients could not undergo the second course because of angitis, catheter occlusion, or worsening of performance status. Among the non-responders, 7 patients switched to sorafenib, whereas 10 patients were ineligible for sorafenib treatment due to liver dysfunction, disease progression, or worsening of performance status. The imaging response by the Response Evaluation Criteria In Solid Tumors to the first course of treatment was CR
Table 1 Background characteristics of patients who received hepatic arterial infusion chemotherapy

\begin{tabular}{|c|c|}
\hline Characteristics & $\begin{array}{l}\text { Median (range) or } \\
\text { patient numbers }\end{array}$ \\
\hline Age (years) & $66(32-88)$ \\
\hline Gender (M/F) & $49 / 6$ \\
\hline ECOG performance status $(0 / 1)$ & $50 / 5$ \\
\hline Etiology (HBV/HCV/others) & $14 / 24 / 17$ \\
\hline Platelet count $\left(/ \mathrm{mm}^{3}\right)$ & $15.6(6.4-41.4)$ \\
\hline Total bilirubin (mg/dL) & $0.9(0.3-1.8)$ \\
\hline Albumin (g/dL) & $3.7(2.7-5.0)$ \\
\hline Prothrombin consumption test (\%) & $78(57.4-118)$ \\
\hline Child-Pugh score $(5 / 6 / 7)$ & $22 / 23 / 10$ \\
\hline Number of liver tumors & $5(1-40)$ \\
\hline Size of liver tumors (mm) & $85(18-170)$ \\
\hline Macroscopic vascular invasion (without/with) & $17 / 38$ \\
\hline$V p(0-2 / 3-4)^{a}$ & $26 / 29$ \\
\hline Vv $(0-1 / 2-3)$ & $46 / 9$ \\
\hline Relative tumor size in the liver $(<50 \% / \geq 50 \%)$ & $47 / 8$ \\
\hline TACE refractory (without/with) & $42 / 13$ \\
\hline Extrahepatic spread (without/with) & $49 / 6$ \\
\hline HCC stage $(I I I / / V a / / V b)^{b}$ & $30 / 22 / 3$ \\
\hline$B C L C$ stage $(B / C)^{c}$ & $18 / 37$ \\
\hline $\operatorname{AFP}(\mathrm{ng} / \mathrm{mL})$ & $1895.2(2.6-529,500)$ \\
\hline $\mathrm{DCP}(\mathrm{mAU} / \mathrm{mL})$ & $3854(24-226,990)$ \\
\hline
\end{tabular}

Abbreviations: AFP alpha-fetoprotein, DCP des-gamma-carboxy prothrombin, ECOG Eastern Cooperative Oncology Group, HBV hepatitis B virus, HCC hepatocellular carcinoma, $H C V$ hepatitis $C$ virus, $V p$ portal invasion, $V_{V}$ venous invasion

${ }^{a} \mathrm{Vp} 0$ through Vp4 indicated no, third branch, second branch (segmental invasion), first branch (branch invasion) and main portal vein invasion, respectively, according to Liver Cancer Study Group of Japan criteria bAccording to the Liver Cancer Group of Japan

'BCLC: Barcelona Clinic Liver Cancer,

in one (1.8\%) patient, PR in 13 (23.6\%), SD in 30 (54.5\%), and PD in $10(18.1 \%)$ patients. SD patients were classified into two groups: 23 patients (41.8\%) had SD with AFP ratio $<1$ or DCP ratio $<1$, whereas $7(12.7 \%)$ had SD with AFP ratio $>1$ and DCP ratio $>1$.

\section{Survival}

Among 55 patients, 27 patients died of HCC; no patients died of other diseases.

In the intent-to-treat population, the 1-year and 2-year survival rates were 64.0 and $48.3 \%$, respectively (Fig. 3a). The median survival time was 19.9 months, and the PFS of the responders to HAIC was 5.0 months (Fig. 3b).

The MST of the responders to HAIC and of the non-responders to the first course of HAIC were 30.5 and 7.7 months, respectively. MST differed significantly between the responders and non-responders $(P<0.001)$. In the responders, the 1-year and 2-year survival rates were 78 


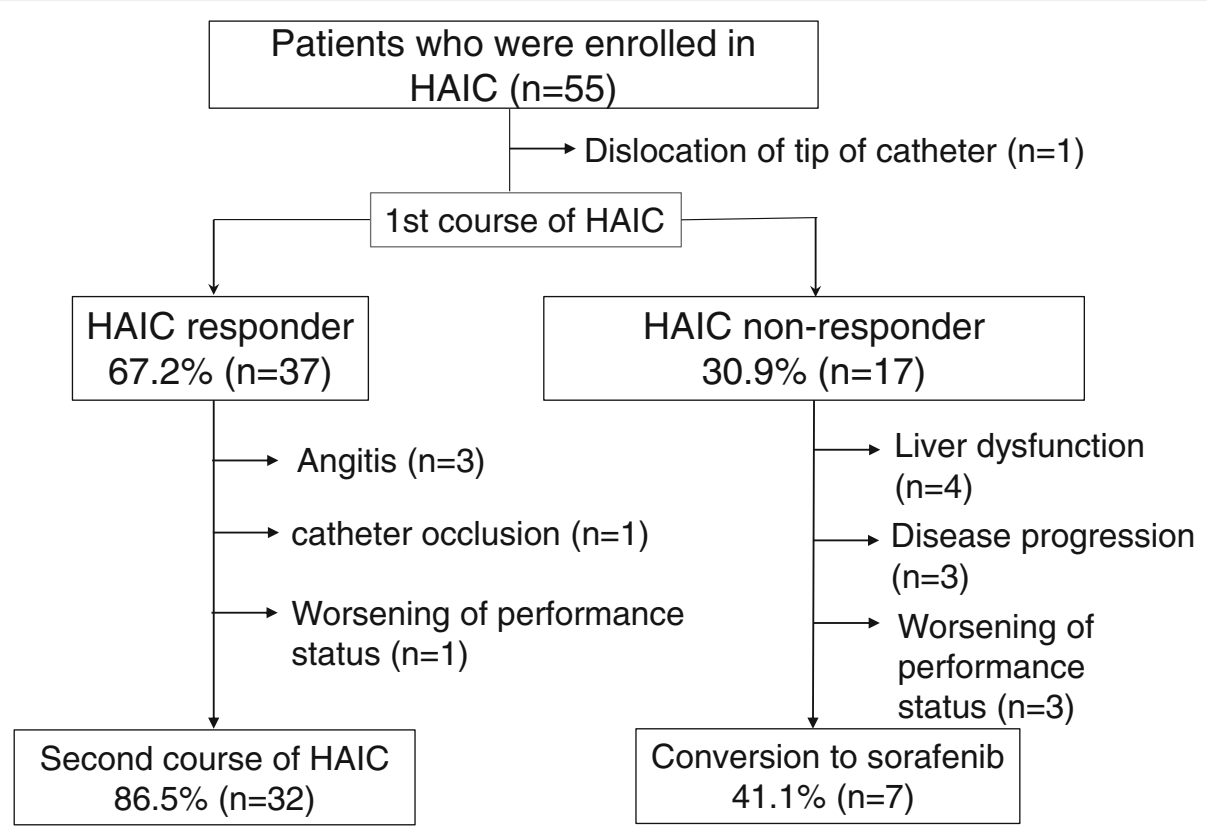

Fig. 2 Patient flow chart. Abbreviations: HAIC hepatic arterial infusion chemotherapy

and $62 \%$, respectively. In the non-responders, the 1-year and 2-year survival rates were 28 and 12\%, respectively (Fig. 4a).

MST differed significantly among the imaging response groups $(P<0.0001): 26.6,30.5,12.0$, and 6.0 months in patients with PR, SD (AFP ratio $<1$ or DCP ratio $<1$ ), $\mathrm{SD}$ (AFP ratio $>1$ and $\mathrm{DCP}$ ratio $>1$ ), and $\mathrm{PD}$, respectively (Fig. 4b).

\section{Safety profile}

Adverse events (AE) during the first course of HAIC are shown in Table 2. The most common AEs were anemia, platelet count decrease, AST/ALT increase and leucocyte count decrease. However, the frequency of $\mathrm{AE} \geq$ grade 3 was $21.8 \%$.

\section{Predictive parameters of efficacy and overall survival}

The univariate analysis identified three parameters that were correlated either significantly or marginally with response: TACE refractory status (without TACE refractory; $P=0.007$ ), and MVI (without MVI; $P=0.018$ ). TACE refractory status and MVI were entered into the multiple logistic regression analysis to identify significant

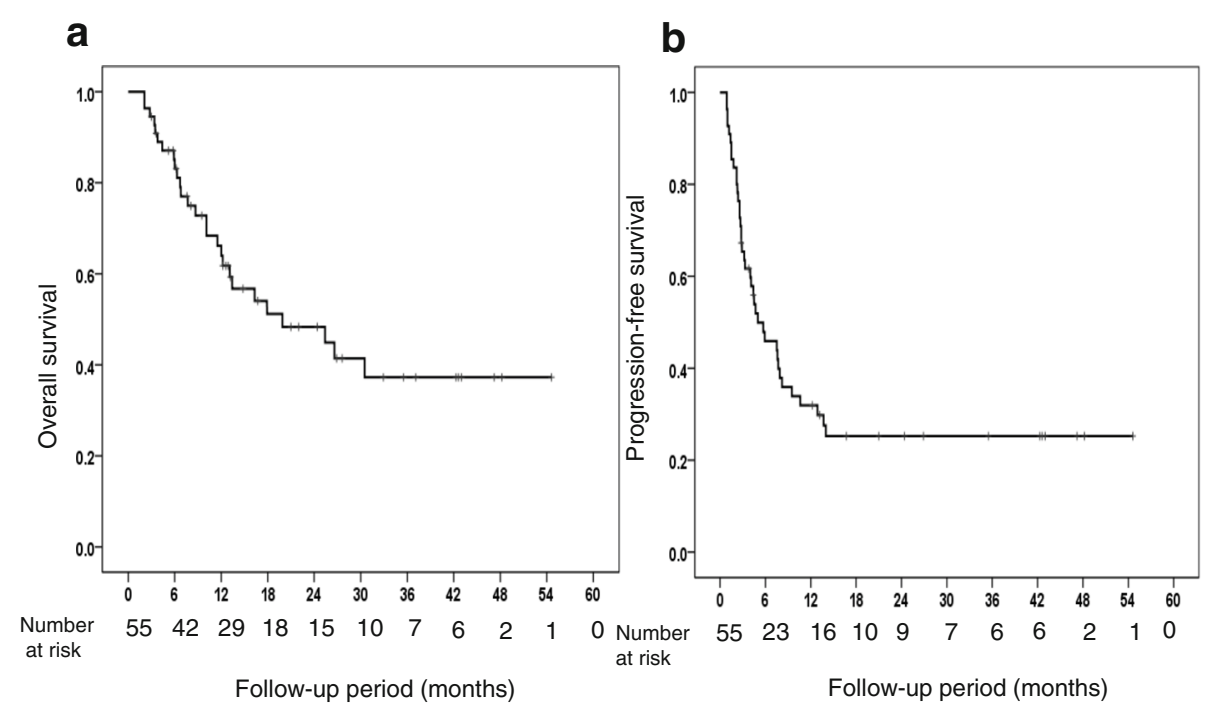

Fig. 3 (a) Overall survival (b) Progression free survival 
a
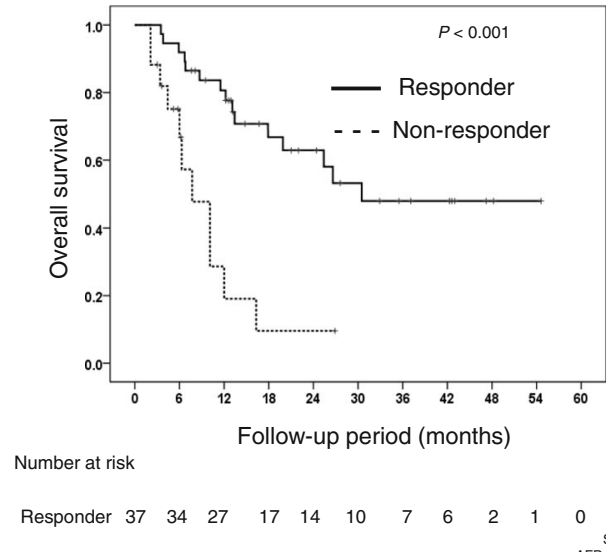

\section{b}

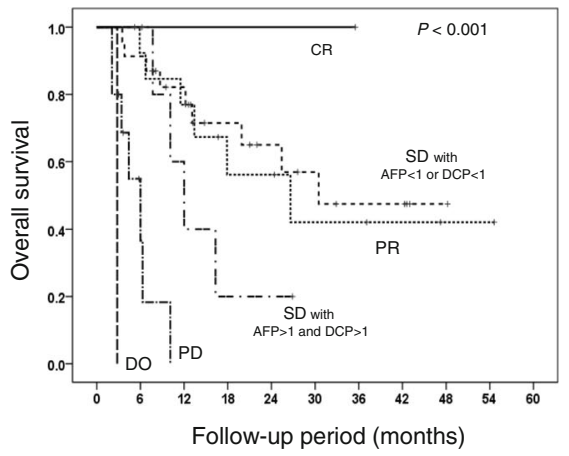

Number at risk

CR 1

$\begin{array}{lllllllllll}\mathrm{PR} & 13 & 12 & 10 & 5 & 5 & 3 & 3 & 2 & 1 & 0\end{array}$

Non-responder $\begin{array}{llllll}17 & 9 & 3 & 1 & 1 & 0\end{array}$

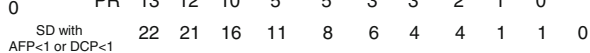

$\begin{array}{ccccccc}\stackrel{\mathrm{SD} \text { with }}{\mathrm{AFP}>1 \text { and } \mathrm{DCP}>1} & 6 & 6 & 3 & 1 & 1 & 0\end{array}$

$\mathrm{PD} \quad 9 \quad 3 \quad 0$

DO $\quad 1 \quad 0$

Fig. 4 (a) Overall survival according to response (b) Overall survival according to responder or non-responder status

independent predictive factors. The multivariate analysis identified the without-TACE refractory stratus as the only significant and independent factor that influenced response (Table 3).

By means of univariate analysis, we then investigated the relationship between survival after the initiation of HAIC treatment and various clinicopathological variables (Table 4). Child-Pugh A, platelet count, DCP and EHS correlated significantly with OS. The above parameters were then entered into a multiple Cox proportional-hazard model analysis. This analysis identified EHS as a significant and independent determinant of survival.

Subgroup analysis was performed according to Child-Pugh status, macroscopic vessel invasion, EHS and TACE refractory status. MST (25 months) of Child-Pugh A patients was significantly longer than that (13 months) of Child-Pugh B patients ( $P=0.0007)$ (Fig. 5a). The MST of patients who had HCC with and without macroscopic vessel invasion were not significantly different: 25.4 months and 16.3 months, respectively (Fig. $5 \mathrm{~b}$ ). The MST of

Table 2 Adverse events associated with the first course of hepatic arterial infusion chemotherapy

\begin{tabular}{|c|c|c|c|c|c|}
\hline No. (\%) & Grade 1 & Grade 2 & Grade 3 & Grade 4 & Total \\
\hline \multicolumn{6}{|l|}{ Clinical } \\
\hline Nausea/Nomiting & $9(17)$ & $2(4)$ & 0 & 0 & $11(20)$ \\
\hline Anorexia & $10(19)$ & $7(13)$ & $1(25)$ & 0 & $18(33)$ \\
\hline Fever & $5(9)$ & $1(25)$ & 0 & 0 & $6(11)$ \\
\hline Pain & $11(20)$ & 0 & 0 & 0 & $11(20)$ \\
\hline Fatigue & $8(15)$ & $1(25)$ & 0 & 0 & $9(17)$ \\
\hline Diarrhea & 0 & $1(25)$ & 0 & 0 & $1(25)$ \\
\hline \multicolumn{6}{|l|}{ Laboratory abnormalities } \\
\hline Leucocyte count decrease & $22(41)$ & $6(11)$ & $1(25)$ & 0 & $29(54)$ \\
\hline Neutrophil count decrease & $10(19)$ & $2(4)$ & 0 & 0 & $12(22)$ \\
\hline Anemia & $26(48)$ & $7(13)$ & $2(4)$ & 0 & $35(65)$ \\
\hline Platelet count decrease & $22(41)$ & $9(17)$ & $4(7)$ & 0 & $35(65)$ \\
\hline AST/ALT increase & $24(44)$ & $4(7)$ & $4(7)$ & 0 & $32(59)$ \\
\hline Creatinine increase & $15(28)$ & 0 & 0 & 0 & $15(28)$ \\
\hline Total & & & $12(21.8)$ & $0(0)$ & \\
\hline
\end{tabular}

Abbreviations: ALT alanine aminotransferase, AST aspartate aminotransferase 
Table 3 Univariate and multivariate analyses of factors associated with response

\begin{tabular}{|c|c|c|c|c|}
\hline \multirow[t]{2}{*}{ Parameters } & \multirow{2}{*}{$\begin{array}{l}\text { Univariate analysis } \\
P \text { value }\end{array}$} & \multicolumn{3}{|c|}{ Multivariate analysis } \\
\hline & & Odds ratio & $95 \% \mathrm{Cl}$ & $P$ value \\
\hline Age $(<65 / \geq 65$ years $)$ & 0.462 & & & \\
\hline Gender (Male/Female) & 0.917 & & & \\
\hline ECOG performance status $(0 / 1)$ & 0.667 & & & \\
\hline Platelet count $\left(<14.9 \times 10^{4} />14.9 \times 10^{4} / \mu \mathrm{L}\right)$ & 0.487 & & & \\
\hline Child-Pugh score (A/B) & 0.736 & & & \\
\hline Diameter of main tumor $(<80 \mathrm{~mm} / \geq 80 \mathrm{~mm})$ & 0.52 & & & \\
\hline Macroscopic vascular invasion (without/with) & 0.018 & & & \\
\hline TACE refractory (without/with) & 0.007 & 5.689 & $1.490-21.724$ & 0.011 \\
\hline $\operatorname{AFP}(<1895 / \geq 1895 \mathrm{ng} / \mathrm{mL})$ & 0.149 & & & \\
\hline $\mathrm{DCP}(<3854 / \geq 3854 \mathrm{mAU} / \mathrm{mL})$ & 0.547 & & & \\
\hline Extrahepatic spread (without/with) & 0.3 & & & \\
\hline
\end{tabular}

Abbreviations: AFP alpha-fetoprotein, DCP des-gamma-carboxy prothrombin, ECOG Eastern Cooperative Oncology Group, MVI macroscopic vascular invasion, TACE transarterial chemoembolization

patients who had HCC without EHS was significantly longer than that of patients who had HCC with EHS (26.6 vs 6.3 months, respectively) $(P<0.001)$ (Fig. 5c). The MST of patients without and with TACE refractory status was not significantly different: 25.4 months and 16.3 months, respectively (Fig. 5d).

\section{Discussion}

We investigated the efficacy of a protocol in which HAIC was selected as the first-line therapy for patients with advanced HCC and sorafenib was selected as the second-line therapy for patients refractory to HAIC. In our study, the 1-year and 2-year survival rates were 64.0 and $48.3 \%$, and the MST was 19.9 months. OS was judged to be favorable with HAIC as first-line therapy for patients with advanced HCC. The rate of AEs (grade $\geq 3: 21.8 \%$ ) was judged to be acceptable by the investigators.

Sorafenib is currently the standard first-line therapy for advanced HCC patients. However, the MST and response rate were almost 10 months and $10 \%$ with sorafenib therapy, respectively. In addition, HAIC is not used as a standard therapy for advanced HCC patients due to the lack of clinical trial data supporting its use.

The primary endpoint of the 1-year survival rate was $64.0 \%$, and the MST was 30.5 months. When we compared our protocol to other treatment protocols for advanced HCC, the 1-year survival rates in the SHARP study and in the Asia-Pacific study of sorafenib monotherapy were 44 and $32 \%$, respectively $[13,14]$. HAIC therapy followed by sorafenib was superior to sorafenib monotherapy. In subgroup analysis of our study, the MST of patients who had

Table 4 Univariate and multivariate analyses for determinants of overall survival

\begin{tabular}{|c|c|c|c|c|}
\hline \multirow[t]{2}{*}{ Parameters } & \multirow{2}{*}{$\begin{array}{l}\text { Univariate analysis } \\
P \text { value }\end{array}$} & \multicolumn{3}{|c|}{ Multivariate analysis } \\
\hline & & Hazard ratio & $95 \% \mathrm{Cl}$ & $P$ value \\
\hline Age $(<65 / \geq 65$ years $)$ & 0.304 & & & \\
\hline Gender (Male/Female) & 0.325 & & & \\
\hline ECOG performance status $(0 / 1)$ & 0.187 & & & \\
\hline Platelet count $\left(<14.9 \times 10^{4} />14.9 \times 10^{4} / \mu \mathrm{L}\right)$ & 0.07 & & & \\
\hline Child-Pugh score (A/B) & 0.008 & & & \\
\hline Diameter of main tumor $(<80 \mathrm{~mm} / \geq 80 \mathrm{~mm})$ & 0.036 & & & \\
\hline Macroscopic vascular invasion (without/with) & 0.646 & & & \\
\hline TACE refractory (without/with) & 0.101 & & & \\
\hline $\operatorname{AFP}(<1895 / \geq 1895 \mathrm{ng} / \mathrm{mL})$ & 0.515 & & & \\
\hline $\mathrm{DCP}(<3854 / \geq 3854 \mathrm{mAU} / \mathrm{mL})$ & 0.055 & & & \\
\hline Extrahepatic spread (without/with) & 0.004 & 3.905 & $1.420-10.736$ & 0.008 \\
\hline
\end{tabular}

Abbreviations: AFP alpha-fetoprotein, DCP des-gamma-carboxy prothrombin, ECOG Eastern Cooperative Oncology Group, MVI macroscopic vascular invasion, TACE transarterial chemoembolization 

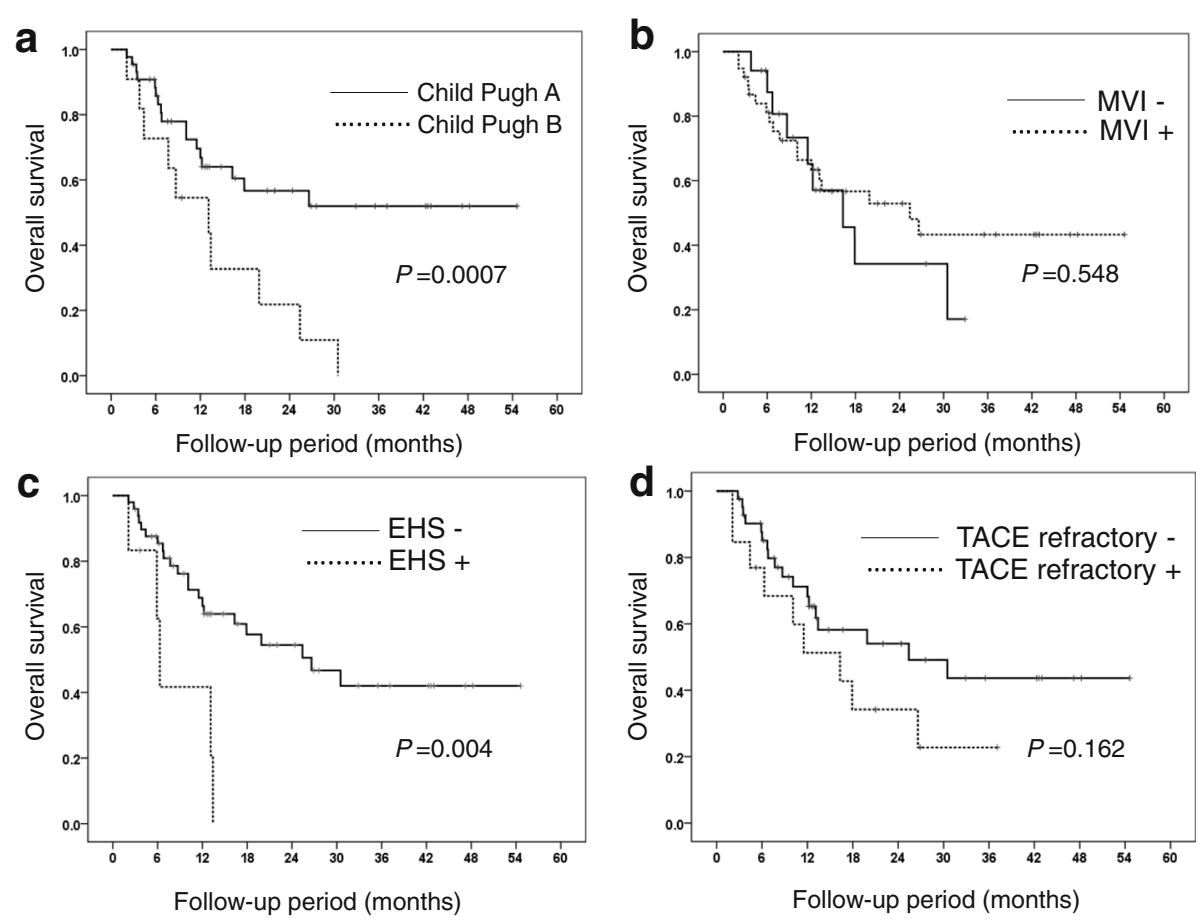

Fig. 5 (a) Overall survival according to Child Pugh grade (b), macroscopic vessel invasion (MVI), (c) extrahepatic spread (EHS), and (d) transcatheter arterial chemoembolization (TACE) refractory

HCC with and without macroscopic vessel invasion was not significantly different: 25.4 months and 16.3 months, respectively (Fig. 5b). The MST of patients who had HCC without EHS was significantly longer than that of patients who had HCC with EHS (26.6 vs 6.3 months, respectively) $(P<0.001)$ (Fig. $5 c)$. Bruix et al. reported that the MST of patients who had $\mathrm{HCC}$ with and without macroscopic vessel invasion were 8.1 months and 14.1 months, respectively, in sub-analysis of the SHARP study [21]. Cheng et al. reported that the MST of patients who had HCC with macroscopic vessel invasion and/or EHS was 5.6 months, and the MST of patients who had HCC without macroscopic vessel invasion or EHS was 14.3 months, respectively, in sub-analysis of the Asia-Pacific trial. Therefore, HAIC therapy followed by sorafenib was superior to sorafenib monotherapy in patients with macroscopic vessel invasion. HAIC therapy followed by sorafenib was not inferior to sorafenib monotherapy in patients with EHS [21]. Furthermore, we compared our protocol to a previous HAIC study. Nouso et al. reported that the 1 -year survival rate of HAIC was $52 \%$ in a nationwide study in Japan [22]. Although, there were few differences between our protocol and Nouso's study, results of HAIC therapy followed by sorafenib in our study was superior to that of the previous HAIC study. The reason for our favorable results could be that we continued HAIC in HAIC responders, who are expected to have good prognoses, and switched to sorafenib therapy in HAIC non-responders, avoiding unnecessary AEs associated with HAIC.

In our study, $21.8 \%$ of patients had $\mathrm{AE} \geq$ grade 3 . Similar to our study, rates of $\mathrm{AE} \geq$ grade 3 were 36 and $23.5 \%$ in the SHARP and Asia-Pacific studies, respectively $[13,14]$. While occlusion of catheter and angitis as HAIC-specific AEs were observed in our study, the rates of HAIC-specific AEs in this study were similar to those of previous studies [11, 12].

Our multivariate analysis identified TACE non-refractory status as the only significant and independent factor that influenced response. In addition, a multiple Cox proportional-hazard model analysis identified lack of EHS as a significant and independent determinant for OS. Retrospective studies have shown similar results. In two studies, OS was significantly longer in those treated with sorafenib compared with HAIC in HCC patients refractory to TACE. A possible reason is that those studies involved shorter duration of HAIC and a need to withdraw the treatment due to stenosis of hepatic artery by catheter therapy, reduced sensitivity to the drug, deterioration of liver function, and appearance of collateral arteries [23, 24]. Another study reported that EHS was a poor prognosis factor in HAIC therapy [25]. Given these results, if our study protocol were to be conducted in patients with TACE non-refractory status and without EHS, favorable results are likely. This protocol should therefore be taken into consideration in the study design of a future clinical trial. 
The study had several limitations: it was a single-arm study with a small sample size and a narrow period of observation. While we need to follow the prognosis over a longer time period, the results of this prospective study show the usefulness of this protocol as a first-line therapy for patients with advanced HCC. Larger comparative studies are necessary to confirm this conclusion.

\section{Conclusion}

We found favorable outcomes in patients with advanced HCC treated with HAIC as first-line therapy. Given the results of this study, this protocol deserves consideration as an optional therapy for advanced HCC patients in the future.

\section{Abbreviations}

AE: Adverse event; CTCAE: Common Terminology Criteria for Adverse Events; HAIC: Hepatic arterial infusion chemotherapy; HCC: Hepatocellular carcinoma; HICS: Hepatic Arterial Infusion Chemotherapy followed by Sorafenib; MST: Median survival time; OS: Overall survival; PFS: Progression-free survival; TACE: Transcatheter arterial chemoembolization

\section{Acknowledgements}

We dedicate this manuscript to Dr Daisuke Miyaki.

\section{Availability of data and materials}

The datasets generated and analyzed during the current study were not approved for public release by the Ethics Review Committee of Hiroshima University but are available from the corresponding author on reasonable request.

\section{Authors' contributions \\ $\mathrm{MH}, \mathrm{TK}$ and $\mathrm{HA}$ were the main authors of the manuscript. They were involved in the conception, design and coordination of the study as well as in data analysis, interpretation of results and drafting of the manuscript. YI, KM, TN, EM, MT, AH, MI, YK, KM, KW, HtK, HsK, TM, YN, TT, HA, YK, KC participated in the collection and analysis of data. KA contributed to assessment of therapy by dynamic computed tomography or magnetic resonance imaging. All authors contributed to the interpretation of data and critically revised the manuscript. All authors read and approved the final manuscript.}

\section{Ethics approval and consent to participate}

This study was prospective and approved by the Ethics Review Committee of the Hiroshima University.

All participants provided written informed consent to participate.

\section{Competing interests}

The authors declare that they have no competing interests.

\section{Publisher's note}

Springer Nature remains neutral with regard to jurisdictional claims in published maps and institutional affiliations.

\footnotetext{
Author details

'Department of Gastroenterology and Metabolism, Institute of Biomedical \& Health Science, Hiroshima University, Hiroshima 734-8551, Japan. ${ }^{2}$ Department of Diagnostic Radiology, Graduate School of Biomedical Sciences, Hiroshima 734-8551, Japan. ${ }^{3}$ Hiroshima City Asa Hospital, Hiroshima, Japan. ${ }^{4}$ Kure Medical Center, Hiroshima, Japan. ${ }^{5}$ Chugoku Rousai Hospital, Hiroshima, Japan. ${ }^{6}$ Mazda Hospital, Hiroshima, Japan. ${ }^{7}$ Onomichi General Hospital, Hiroshima, Japan. ${ }^{8}$ Liver Research Project Center, Hiroshima University, Hiroshima, Japan. 'Laboratory for Digestive Diseases, RIKEN Center for Integrative Medical Sciences, Hiroshima, Japan.
}

Received: 18 October 2017 Accepted: 18 May 2018

Published online: 04 June 2018

\section{References}

1. Ferlay J, Shin HR, Bray F, Forman D, Mathers C, Parkin DM. Estimates of worldwide burden of cancer in 2008: GLOBOCAN 2008. Int J Cancer. 2010;127(12):2893-917.

2. Kamangar F, Dores GM, Anderson WF. Patterns of cancer incidence, mortality, and prevalence across five continents: defining priorities to reduce cancer disparities in different geographic regions of the world. J Clin Oncol. 2006;24(14):2137-50.

3. Shaw C, Blakely T, Sarfati D, Fawcett J, Peace J. Trends in colorectal cancer mortality by ethnicity and socio-economic position in New Zealand, 198199: one country, many stories. Aust N Z J Public Health. 2006;30(1):64-70.

4. Livraghi T, Giorgio A, Marin G, Salmi A, de Sio I, Bolondi L, Pompili M, Brunello F, Lazzaroni S, Torzilli G, et al. Hepatocellular carcinoma and cirrhosis in 746 patients: long-term results of percutaneous ethanol injection. Radiology. 1995;197(1):101-8.

5. Kamada K, Kitamoto M, Aikata H, Kawakami Y, Kono H, Imamura M, Nakanishi T, Chayama K. Combination of transcatheter arterial chemoembolization using cisplatin-lipiodol suspension and percutaneous ethanol injection for treatment of advanced small hepatocellular carcinoma. Am J Surg. 2002;184(3):284-90.

6. Livraghi T, Goldberg SN, Lazzaroni S, Meloni F, Solbiati L, Gazelle GS. Small hepatocellular carcinoma: treatment with radio-frequency ablation versus ethanol injection. Radiology. 1999;210(3):655-61.

7. Goldstein HM, Wallace S, Anderson JH, Bree RL, Gianturco C. Transcatheter occlusion of abdominal tumors. Radiology. 1976;120(3):539-45.

8. Chuang VP, Wallace S. Hepatic artery embolization in the treatment of hepatic neoplasms. Radiology. 1981;140(1):51-8.

9. Stehlin JS Jr, de Ipolyi PD, Greeff PJ, McGaff CJ Jr, Davis BR, McNary L. Treatment of cancer of the liver. Twenty years' experience with infusion and resection in 414 patients. Ann Surg. 1988;208(1):23-35.

10. Doci R, Bignami P, Bozzetti F, Bonfanti G, Audisio R, Colombo M, Gennari L. Intrahepatic chemotherapy for unresectable hepatocellular carcinoma. Cancer. 1988;61(10):1983-7.

11. Ando E, Tanaka M, Yamashita F, Kuromatsu R, Yutani S, Fukumori K, Sumie S, Yano Y, Okuda K, Sata M. Hepatic arterial infusion chemotherapy for advanced hepatocellular carcinoma with portal vein tumor thrombosis: analysis of 48 cases. Cancer. 2002;95(3):588-95.

12. Obi S, Yoshida H, Toune R, Unuma T, Kanda M, Sato S, Tateishi R, Teratani T, Shiina S, Omata M. Combination therapy of intraarterial 5-fluorouracil and systemic interferon-alpha for advanced hepatocellular carcinoma with portal venous invasion. Cancer. 2006;106(9):1990-7.

13. Llovet JM, Ricci S, Mazzaferro V, Hilgard P, Gane E, Blanc JF, de Oliveira AC, Santoro A, Raoul JL, Forner A, et al. Sorafenib in advanced hepatocellular carcinoma. N Engl J Med. 2008;359(4):378-90.

14. Cheng AL, Kang YK, Chen Z, Tsao CJ, Qin S, Kim JS, Luo R, Feng J, Ye S, Yang TS, et al. Efficacy and safety of sorafenib in patients in the Asia-Pacific region with advanced hepatocellular carcinoma: a phase III randomised, double-blind, placebo-controlled trial. Lancet Oncol. 2009;10(1):25-34.

15. Uka K, Aikata H, Takaki S, Miki D, Kawaoka T, Jeong SC, Takahashi S, Toyota $\mathrm{N}$, Ito K, Chayama K. Pretreatment predictor of response, time to progression, and survival to intraarterial 5-fluorouracil/interferon combination therapy in patients with advanced hepatocellular carcinoma. J Gastroenterol. 2007:42(10):845-53.

16. Uka K, Aikata H, Takaki S, Miki D, Jeong SC, Hiramatsu A, Kodama H, Shirakawa H, Kawakami Y, Takahashi S, et al. Similar effects of recombinant interferon-alpha-2b and natural interferon-alpha when combined with intraarterial 5-fluorouracil for the treatment of advanced hepatocellular carcinoma. Liver Int. 2007;27(9):1209-16.

17. Katamura Y, Aikata H, Takaki S, Azakami T, Kawaoka T, Waki K, Hiramatsu A, Kawakami Y, Takahashi S, Kenjo M, et al. Intra-arterial 5-fluorouracil/ interferon combination therapy for advanced hepatocellular carcinoma with or without three-dimensional conformal radiotherapy for portal vein tumor thrombosis. J Gastroenterol. 2009;44(5):492-502.

18. Miyaki D, Aikata H, Honda Y, Naeshiro N, Nakahara T, Tanaka M, Nagaoki Y, Kawaoka T, Takaki S, Waki K, et al. Hepatic arterial infusion chemotherapy for advanced hepatocellular carcinoma according to child-Pugh classification. J Gastroenterol Hepatol. 2012;27(12):1850-7. 
19. Gomez-Campdera FJ, Anaya F, Robles R, Rengel-Aranda MA, Valderrabano F. Renal transplantation from anencephalic donors. Nephron. 1989;52(1):98-9.

20. Miyaki D, Kawaoka T, Aikata H, Kan H, Fujino H, Fukuhara T, Kobayashi T, Naeshiro N, Honda Y, Tsuge M, et al. Evaluation of early response to hepatic arterial infusion chemotherapy in patients with advanced hepatocellular carcinoma using the combination of RECIST and tumor markers. J Gastroenterol Hepatol. 2015;30(4):726-32.

21. Bruix J, Raoul JL, Sherman M, Mazzaferro V, Bolondi L, Craxi A, Galle PR, Santoro A, Beaugrand M, Sangiovanni A, et al. Efficacy and safety of sorafenib in patients with advanced hepatocellular carcinoma: subanalyses of a phase III trial. J Hepatol. 2012;57(4):821-9.

22. Nouso K, Miyahara K, Uchida D, Kuwaki K, Izumi N, Omata M, Ichida T, Kudo M, Ku Y, Kokudo N, et al. Effect of hepatic arterial infusion chemotherapy of 5 -fluorouracil and cisplatin for advanced hepatocellular carcinoma in the Nationwide survey of primary liver Cancer in Japan. Br J Cancer. 2013;109(7): 1904-7.

23. Ikeda M, Mitsunaga S, Shimizu S, Ohno I, Takahashi H, Okuyama H Kuwahara A, Kondo S, Morizane C, Ueno H, et al. Efficacy of sorafenib in patients with hepatocellular carcinoma refractory to transcatheter arterial chemoembolization. J Gastroenterol. 2014;49(5):932-40.

24. Hatooka M, Kawaoka T, Aikata H, Morio K, Kobayashi T, Hiramatsu A, Imamura M, Kawakami Y, Murakami E, Waki K, et al. Comparison of outcome of hepatic arterial infusion chemotherapy and Sorafenib in patients with hepatocellular carcinoma refractory to Transcatheter arterial chemoembolization. Anticancer Res. 2016;36(7):3523-9.

25. Katamura Y, Aikata H, Kimura Y, Kawaoka T, Takaki S, Waki K, Hiramatsu A, Kawakami Y, Takahashi S, Ishikawa M, et al. Intra-arterial 5-fluorouracil/ interferon combination therapy for hepatocellular carcinoma with portal vein tumor thrombosis and extrahepatic metastases. J Gastroenterol Hepatol. 2010:25(6):1117-22.

\section{Ready to submit your research? Choose BMC and benefit from:}

- fast, convenient online submission

- thorough peer review by experienced researchers in your field

- rapid publication on acceptance

- support for research data, including large and complex data types

- gold Open Access which fosters wider collaboration and increased citations - maximum visibility for your research: over $100 \mathrm{M}$ website views per year

At BMC, research is always in progress.

Learn more biomedcentral.com/submissions 